\title{
SOME I-CONVERGENT SEQUENCE SPACES OF FUZZY NUMBERS DEFINED BY INFINITE MATRIX
}

\author{
Ekrem Savaş \\ Department of Mathematics, Istanbul Commerce University, \\ Üsküdar-İstanbul, Turkey \\ ekremsavas@yahoo.com
}

\begin{abstract}
In this paper we introduce and study some new sequence spaces of fuzzy numbers defined by $I$-convergence using the sequences of Orlicz functions, infinite matrix. We study some basic topological and algebraic properties of these spaces. Also we investigate the relations related to these spaces.
\end{abstract}

Key Words- Ideal, I -convergent, infinite matrix, Orlicz function, fuzzy number.

\section{INTRODUCTION}

The theory of sequence of fuzzy numbers was first introduced by Matloka [10]. Matloka introduced bounded and convergent sequence of fuzzy numbers and studied some of their properties and showed that every convergent sequence of fuzzy numbers is bounded. Nanda [12] studied the sequence of fuzzy numbers and showed that the set of all convergent sequence of fuzzy numbers forms a complete metric space. J. S. Kwon [8] introduced the definition of strongly $p$-Cesaro summability of sequence of fuzzy numbers. Savas [17] introduced and discussed double convergent sequence of fuzzy numbers and showed that the set of all double convergent sequence of fuzzy numbers is complete. Savas [24] studied some equivalent alternative conditions for a sequence of fuzzy numbers to be statistically Cauchy and he continued to study the statistical convergence in [22, 25]. Recently, Mursaleen and Basarir [11] introduced and studied some new sequence space of fuzzy numbers generated by non-negative regular matrix. Also Savas and Mursaleen [21] defined statistically convergent and statistically Cauchy for double sequence of fuzzy numbers.

Different classes of sequence of fuzzy real numbers have been discussed by Nuray and Savas [13], Altinok et al. [1], Hazarika and Savas [2], Kumar and Kumar [7], Savas ( [22], [23]), Savas and Mursaleen [21], Joong-Sung [8] and many others.

The notion of I-convergence initially introduced by Kostyrko et al. [6]. More investigations in this direction and more applications of ideals can be found in $[18,19$, $20,30,31,32]$ where many important references can be found.

Let $X$ be a non-empty set, then a family of sets $I \subset 2^{X}$ (the class of all subsets of $X$ ) is called an ideal if and only if for each $A, B \in I$, we have $A \cup B \in I$ and for each $A \in I$ and each $B \subset A$, we have $B \in I$. A non-empty family of sets $F \subset 2^{X}$ is a filter on $X$ if and only if $\varnothing \notin F$, for each $A, B \in F$, we have $A \cap B \in F$ and each $A \in F$ and each $A \subset B$, we have $B \in F$. An ideal $I$ is called non-trivial ideal if $I \neq \varnothing \mathrm{I}$ and $X \notin I$.Clearly 
$I \subset 2^{X}$ is a non-trivial ideal if and only if $F=F(I)=\{X-A: A \in I\}$ is a filter on $X$. A non-trivial ideal $I \subset 2^{X}$ is called admissible if and only if $\{\{x\}: x \in X\} \subset I$. A non-trivial ideal $I$ is maximal if there cannot exists any non-trivial ideal $J \neq I$ containing $I$ as a subset. Further details on ideals of $2^{X}$ can be found in Kostyrko et al. [22].

Recall in [5] that an Orlicz function $M$ is continuous, convex, nondecreasing function such that $M(0)=0$ and $M(x)>0$ for $x>0$ and $M(x) \rightarrow \infty$ as $x \rightarrow \infty$. If convexity of Orlicz function is replaced by $M(x+y)=M(x)+M(y)$ then this function is called the modulus function and characterized by Ruckle [16]. An Orlicz function $M$ is said to satisfy $\Delta_{2}$-condition for all values of $u$, if there exists $K>0$ such that $M(2 u) \leq K M(u)$, $u \geq 0$. as follows:

Lindenstrauss and Tzafriri [9] studied some Orlicz type sequence spaces defined

$$
\ell_{M}=\left\{\left(x_{k}\right) \in w: \sum_{k=1}^{\infty} M\left(\frac{\left|x_{k}\right|}{\rho}\right)<\infty, \text { for some } \rho>0\right\} .
$$

The space $\ell_{M}$ with the norm

$$
\|x\|=\inf \left\{\rho>0: \sum_{k=1}^{\infty} M\left(\frac{\left|x_{k}\right|}{\rho}\right) \leq 1\right\} .
$$

becomes a Banach space which is called an Orlicz sequence space. The space $\ell_{M}$ is closely related to the space $\ell_{p}$ which is an Orlicz sequence space with $M(t)=|t|^{p}$, for $1 \leq p<\infty$.

The following well-known inequality will be used throughout the article. Let $p=\left(p_{k}\right)$ be any sequence of positive real numbers with $0 \leq p_{k} \leq \sup _{k} p_{k}=H, D=\max \{1$, $2^{H-1}$ \}then

$$
\left|a_{k}+b_{k}\right|^{p_{k}} \leq D\left(\left|a_{k}\right|^{p_{k}}+\left|b_{k}\right|^{p_{k}}\right)
$$

for all $k \in N$ and $a_{k}, b_{k} \in C$. Also $\left|a_{k}\right|^{p_{k}} \leq \max \left\{1,|a|^{H}\right\}$ for all $a \in C$.

In the later stage different classes of Orlicz sequence spaces were introduced and studied by Parashar and Choudhary [14], Savas ([26]-[29]) and many others.

Throughout the article $w^{F}$ denote the class of all fuzzy real-valued sequence space. Also $N$ and $R$ denote the set of positive integers and set of real numbers respectively.

In this paper, we study some new sequence spaces of fuzzy numbers defined by using $I$-convergence, the sequence of Orlicz functions and an infinite matrix. We establish inclusion relations between the sequence spaces $w^{I(F)}[A, M, p], w^{I(F)}[A, M, p]_{0}$, $w^{F}[A, M, p]_{\infty}$ and $w^{I(F)}[A, M, p]_{\infty}$ where $p=\left(p_{k}\right)$ denote the sequence of positive real numbers for all $n \in N$ and $M=\left(M_{k}\right)$ be a sequence of Orlicz functions. 


\section{DEFINITIONS AND NOTATIONS}

Before continuing with this paper we present a few definitions and preliminaries.

Given any interval $A$, we shall denote its end points by $\underline{A}, \bar{A}$ and by $D$ set of all closed bounded intervals on real line $R$ i.e., $D=\{A \subset R: A=[\underline{A}, \bar{A}]\}$. For $A, B \in D$ we define $A \leq B$ if and only if $\underline{A} \leq \underline{B}$ and $\bar{A} \leq \bar{B}$ and

$d(A, B)=\max \{|\underline{A}-\underline{B}|,|\bar{A}-\bar{B}|\}$.

It is easy to see that $d$ defines a Hausdorff metric on $D$ and $(D, d)$ is a complete metric space. Also $\leq$ partial order on $D$.

A fuzzy number is a function $X$ from $R$ to $[0,1]$ which satisfying the following conditions (i) $X$ is normal, i.e. there exists an $x_{0} \in R$ such that $X\left(x_{0}\right)=1$; (ii) $X$ is fuzzy convex, i.e. for any $x, y \in R$ and $\lambda \in[0,1], X(\lambda x+(1-\lambda) y) \geq \min \{X(x), Y(y)\}$; (iii) $X$ is upper semi continuous; (iv) The closure of the set $\{x \in R: X(x)>0\}$, denoted by $X^{0}$ is compact.

The properties (i) to (iv) imply that for each $\alpha \in[0,1]$, the $\alpha$-level set $X^{\alpha}=\{x \in R$ : $X(x)>\alpha\}=\left[\underline{X}^{\alpha}, \bar{X}^{\alpha}\right]$ is a non empty compact convex subset of $R$. Let $L(R)$ denotes the set of all fuzzy numbers. Define a map $\bar{d}: L(R) \times L(R) \rightarrow R$ by $\bar{d}(x, y)=\sup _{\alpha \in[0,1]} d\left(X^{\alpha}, Y^{\alpha}\right)$. Puri and Ralescu [15] proved that $(L(R), \bar{d})$ is a complete metric space.

For $X, Y \in L(R)$, we define $X \leq Y$ if and only if $\underline{X}^{\alpha} \leq \underline{Y}^{\alpha}$ and $\bar{X}^{\alpha}, \bar{Y}^{\alpha}$ for each $\alpha \in[0$, 1], we say that $X<Y$ if $X \leq Y$ and there exist $\alpha_{0} \in[0,1]$ such that $\underline{X}^{\alpha_{0}} \leq \underline{Y}^{\alpha_{0}}$ or $\bar{X}^{\alpha_{0}}, \bar{Y}^{\alpha_{0}}$. The fuzzy number $X$ and $Y$ are said to be incomparable if neither $X \leq Y$ nor $Y \leq X$.

For any $X, Y, Z \in L(R)$, the linear structure of $L(R)$ induced addition $X+Y$ and scalar multiplication $\lambda X, \lambda \in R$, in terms of $\alpha$-level sets, by $[\mathrm{X}+\mathrm{Y}]^{\alpha}=[\mathrm{X}]^{\alpha}+[\mathrm{Y}]^{\alpha}$ and $[\lambda X]^{\alpha}=\lambda[X]^{\alpha}$ for each $\alpha \in[0,1]$.

Proposition 1.1. If $\bar{d}$ is a translation invariant metric on $L(R)$ then

(i) $\bar{d}(\mathrm{X}+\mathrm{Z}, 0) \leq \bar{d}(\mathrm{X}, 0)+\bar{d}(\mathrm{Y}, 0)$,

(ii) $\bar{d}(\lambda \mathrm{X}, 0) \leq|\lambda| \bar{d}(\mathrm{X}, 0),|\lambda|>1$.

A sequence $X=\left(X_{k}\right)$ of fuzzy numbers is said to converge to a fuzzy number $X_{0}$ if for every $\varepsilon>0$, there exists a positive integer $n_{0}$ such that $\bar{d}\left(X_{k}, X_{0}\right)<\varepsilon$ for all $n \geq n_{0}$.A sequence $X=\left(X_{k}\right)$ of fuzzy numbers is said to be bounded if the set $\left\{X_{k}: k \in N\right\}$ of fuzzy numbers is bounded.

A sequence $X=\left(X_{k}\right)$ of fuzzy numbers is said to be I-convergent to a fuzzy number $X_{0}$ if for each $\varepsilon>0$ such that

$$
A=\left\{k \in N: \bar{d}\left(X_{k}, X_{0}\right) \geq \varepsilon\right\} \in I .
$$

The fuzzy number $X_{0}$ is called I-limit of the sequence $\left(X_{k}\right)$ of fuzzy numbers and we write $I-\lim X_{k}=X_{0}$.

A sequence $X=\left(X_{k}\right)$ of fuzzy numbers is said to be I-bounded if there exists $M>0$ such that

$\left\{k \in N: \bar{d}\left(X_{k}, 0\right)>M\right\} \in I$.

Let $E_{F}$ be denote the sequence space of fuzzy numbers. 
A sequence space $E_{F}$ is said to be solid (or normal) if $\left(Y_{k}\right) \in E_{F}$ whenever $\left(X_{k}\right) \in E_{F}$ and $\left|Y_{k}\right| \leq\left|X_{k}\right|$ for all $k \in N$.

A sequence space $E_{F}$ is said to be monotone if $E_{F}$ contains the canonical preimages of all its step spaces.

Example 2.1. If we take $I=I_{f}=\{A \subseteq N: A$ is a finite subset $\}$. Then $I_{f}$ is a nontrivial admissible ideal of $N$ and the corresponding convergence coincide with the usual convergence.

Example 2.2. If we take $I=I_{\delta}=\{A \subseteq N: \delta(A)=0\}$ where $\delta(A)$ denote the asymptotic density of the set $A$. Then $I_{\delta}$ is a non-trivial admissible ideal of $N$ and the corresponding convergence coincide with the statistical convergence.

Lemma 2.1. $A$ sequence space $E_{F}$ is normal implies $E_{F}$ is monotone. (For the crisp set case, one may refer to Kamthan and Gupta (see, [4]).

\section{SOME NEW SEQUENCE SPACES OF FUZZY NUMBERS}

In this section, using the sequence of Orlicz functions, an infinite matrix and $I$ convergence; we introduced the following new sequence spaces and examine some properties of the resulting sequence spaces. Let $I$ be an admissible ideal of $N$ and let $p=\left(p_{k}\right)$ bea sequence of positive real numbers for all $k \in N$, and $A=\left(a_{n k}\right)$ an infinite matrix. Let $M=\left(M_{k}\right)$ be a sequence Orlicz functions and $X=\left(X_{k}\right)$ be a sequence of fuzzy numbers, we define the following new sequence spaces:

$$
\begin{aligned}
& w^{I(F)}[A, M, p]=\left\{\left(X_{k}\right) \in w^{F}: \forall \boldsymbol{\varepsilon}>0,\left\{n \in N: \sum_{k=1}^{\infty} a_{n k}\left[M_{k}\left(\frac{\bar{d}\left(X_{k}, X_{0}\right)}{\rho}\right)\right]^{p_{k}} \geq \boldsymbol{\varepsilon}\right\} \in I, \text { for some } \boldsymbol{\rho}>0 \text { and } X_{0} \in L(R)\right\}, \\
& w^{I(F)}[A, M, p]_{0}=\left\{\left(X_{k}\right) \in w^{F}: \forall \boldsymbol{\varepsilon}>0,\left\{n \in N: \sum_{k=1}^{\infty} a_{n k}\left[M_{k}\left(\frac{\bar{d}\left(X_{k}, \overline{0}\right)}{\rho}\right)\right]^{p_{k}} \geq \boldsymbol{\varepsilon}\right\} \in I, \text { for some } \boldsymbol{\rho}>0\right\}, \\
& w^{F}[A, M, p]_{\infty}=\left\{\left(X_{k}\right) \in w^{F}: \sup _{n} \sum_{k=1}^{\infty} a_{n k}\left[M_{k}\left(\frac{\bar{d}\left(X_{k}, \overline{0}\right)}{\rho}\right)\right]^{p_{k}}<\infty, \text { for some } \boldsymbol{\rho}>0\right\},
\end{aligned}
$$

and

$$
w^{I(F)}[A, M, p]_{\infty}=\left\{\left(X_{k}\right) \in w^{F}: \exists K>0 \text { s.t. },\left\{n \in N: \sum_{k=1}^{\infty} a_{n k}\left[M_{k}\left(\frac{\bar{d}\left(X_{k}, X_{0}\right)}{\rho}\right)\right]^{p_{k}} \geq K\right\} \in I, \text { for some } \boldsymbol{\rho}>0\right\},
$$

Let us consider a few special cases of the above sets.

(i) If $M_{k}(x)=x$ for all $k \in N$, then the above classes of sequences are denoted by $w^{I(F)}[A, p], w^{I(F)}[A, p]_{0}, w^{F}[A, p]_{\infty}$, and $w^{I(F)}[A, p]_{\infty}$, respectively. $M]_{0}$,

(iii) If $p=\left(p_{k}\right)=(1,1,1, \ldots)$, then we denote the above spaces by $w^{I(F)}[A, M], w^{I(F)}[A$, 
$w^{F}[A, M]_{\infty}$, and $w^{I(F)}[A, M]_{\infty}$.

(iv) If we take $A=(C, 1)$,i.e., the Cesaro matrix, then the above classes of sequences are denoted by $w^{I(F)}[w, M, p], w^{I(F)}[w, M, p]_{0}, w^{F}[w, M, p]_{\infty}$, and $w^{I(F)}[\mathrm{w}, M, p]_{\infty}$, respectively.

v) If we take $A=\left(a_{n k}\right)$ is a de la Valeepoussin mean, i.e.,

$$
a_{n k}=\left\{\begin{array}{ccc}
\frac{1}{\lambda_{n}} & ; & \text { if } k \in I_{n}=\left[n-\lambda_{n}+1, n\right] \\
0 & ; & \text { otherwise }
\end{array}\right\}
$$

where $\left(\lambda_{n}\right)$ is a non-decreasing sequence of positive numbers tending to $\infty$ and $\lambda_{n+1} \leq \lambda_{n}+1, \lambda_{l}=1$, then the above classes of sequences are denoted by $w_{\lambda}{ }^{I(F)}[M, p]$, $w_{\lambda}{ }^{I(F)}[M, p]_{0}, w_{\lambda}{ }^{F}[M, p]_{\infty}$, and $w_{\lambda}^{I(F)}[M, p]_{\infty}$, respectively.

(vi) By a lacunary $\theta=\left(k_{r}\right) ; r=0,1,2, \ldots$ where $k_{0}=0$, we shall mean an increasing sequence of non-negative integers with $k_{r}-k_{r-1}$ as $r \rightarrow \infty$. The intervals determined by $\theta$ will be denoted by $I_{r}=\left(k_{r-1}, k_{r}\right]$ and $h_{r}=k_{r}-k_{r-1}$. As a final illustration let

$$
a_{n k}=\left\{\begin{array}{ccc}
\frac{1}{h_{r}} & ; & \text { if } k_{r-1}<k<k_{r}, \\
0 & ; & \text { otherwise }
\end{array}\right\}
$$

Then we denote the above classes of sequences by $w_{\theta}^{I(F)}[\mathrm{A}, M, p], w_{\theta}{ }^{I(F)}[M, p]_{0}$, $w_{\theta}{ }^{F}[M, p]_{\infty}$, and $w_{\theta}{ }^{I(F)}[M, p]_{\infty}$, respectively.

(vii) If $I=I_{f}$, then we obtain

$$
\begin{aligned}
& w^{F}[A, M, p]=\left\{\left(X_{k}\right) \in w^{F}: \lim _{n \rightarrow \infty} \sum_{k=1}^{\infty} a_{n k}\left[M_{k}\left(\frac{\bar{d}\left(X_{k}, X_{0}\right)}{\rho}\right)\right]^{p_{k}}=0, \text { for some } \rho>0 \text { and } X_{0} \in L(R)\right\}, \\
& w^{F}[A, M, p]_{0}=\left\{\left(X_{k}\right) \in w^{F}: \lim _{n \rightarrow \infty} \sum_{k=1}^{\infty} a_{n k}\left[M_{k}\left(\frac{\bar{d}\left(X_{k}, X_{0}\right)}{\rho}\right)\right]^{p_{k}}=0, \text { for some } \rho>0\right\}, \\
& w^{F}[A, M, p]_{\infty}=\left\{\left(X_{k}\right) \in w^{F}: \sup _{n} \sum_{k=1}^{\infty} a_{n k}\left[M_{k}\left(\frac{\bar{d}\left(X_{k}, \overline{0}\right)}{\rho}\right)\right]^{p_{k}}<\infty, \text { for some } \boldsymbol{\rho}>0\right\},
\end{aligned}
$$

(viii) If $I=I_{\delta}$ is an admissible ideal of $N$, then we have

$$
\begin{aligned}
& w^{I(F)}\left[A, M, \Delta^{m}, p\right]=\left\{\left(X_{k}\right) \in w^{F}: \forall \varepsilon>0,\left\{n \in N: \sum_{k=1}^{\infty} a_{n k}\left[M_{k}\left(\frac{\bar{d}\left(X_{k}, X_{0}\right)}{\rho}\right)\right]^{p_{k}} \geq \varepsilon\right\} \in I_{\delta}, \text { for some } \rho>0 \text { and } X_{0} \in L(R)\right\}, \\
& w^{I(F)}\left[A, M, \Delta^{m}, p\right]_{0}=\left\{\left(X_{k}\right) \in w^{F}: \forall \varepsilon>0,\left\{n \in N: \sum_{k=1}^{\infty} a_{n k}\left[M_{k}\left(\frac{\bar{d}\left(X_{k}, \overline{0}\right)}{\rho}\right)\right]^{p_{k}} \geq \varepsilon\right\} \in I_{\delta}, \text { for some } \rho>0\right\},
\end{aligned}
$$

and 
$w^{I(F)}\left[A, M, \Delta^{m}, p\right]_{\infty}=\left\{\left(X_{k}\right) \in w^{F}: \exists K>0\right.$ s.t. $\left\{n \in N: \sum_{k=1}^{\infty} a_{n k}\left[M_{k}\left(\frac{\bar{d}\left(X_{k}, \overline{0}\right)}{\rho}\right)\right]^{p_{k}} \geq K\right\} \in I_{\delta}$, for some $\left.\rho>0\right\}$, If $X=\left(X_{k}\right) \in w^{F}[A, M, p]$ then we say that $X=\left(X_{k}\right)$ is strongly $(p)$ - Cesaro convergent with respect to the sequence of Orlicz functions $M$.

\section{MAIN RESULTS}

In this section, we examine the basic topological and algebraic properties of the new sequence spaces and obtain the inclusion relation related to these spaces.

Theorem 4.1. Let $\left(p_{k}\right)$ be a bounded sequence. Then the sequence spaces $w^{I(F)}[A, M, p]$, $w^{I(F)}[A, M, p]_{0}, w^{I(F)}[A, M, p]_{\infty}$ are linear spaces.

Proof. We shall prove the result for the space $w_{\theta}{ }^{I(F)}[\mathrm{A}, M, p]_{0}$ only and the others can be proved in similar way.

Let $X=\left(X_{k}\right)$ and $Y=\left(Y_{k}\right)$ be two elements in $w_{\theta}{ }^{I(F)}[\mathrm{A}, M, p]_{0}$. Then there exist $\rho_{1}>0$ and $\rho_{2}>0$ such that

$$
A_{\frac{\varepsilon}{2}}=\left\{r \in N: \sum_{k=1}^{\infty} a_{n k}\left[M_{k}\left(\frac{\bar{d}\left(X_{k}, \overline{0}\right)}{\rho_{1}}\right)\right]^{p_{k}} \geq \frac{\varepsilon}{2}\right\} \in I
$$

and

$$
B_{\frac{\varepsilon}{2}}=\left\{r \in N: \sum_{k=1}^{\infty} a_{n k}\left[M_{k}\left(\frac{\bar{d}\left(Y_{k}, \overline{0}\right)}{\rho_{2}}\right)\right]^{p_{k}} \geq \frac{\varepsilon}{2}\right\} \in I
$$

Let $\alpha, \beta$ be two scalars. By the continuity of the function $\mathrm{M}=\left(M_{k}\right)$ the following inequality holds:

$$
\begin{aligned}
& \sum_{k=1}^{\infty} a_{n k}\left[M_{k}\left(\frac{\bar{d}\left(\boldsymbol{\alpha} X_{k}+\boldsymbol{\beta} Y_{k}, \overline{0}\right)}{|\boldsymbol{\alpha}| \boldsymbol{\rho}_{1}+|\boldsymbol{\beta}| \boldsymbol{\rho}_{2}}\right)\right]^{p_{k}} \\
& \leq D \sum_{k=1}^{\infty} a_{n k}\left[\frac{|\boldsymbol{\alpha}|}{|\boldsymbol{\alpha}| \boldsymbol{\rho}_{1}+|\boldsymbol{\beta}| \boldsymbol{\rho}_{2}} M_{k}\left(\frac{\bar{d}\left(X_{k}, \overline{0}\right)}{\rho_{1}}\right)\right]^{p_{k}}+\leq D \sum_{k=1}^{\infty} a_{n k}\left[\frac{|\boldsymbol{\beta}|}{|\boldsymbol{\alpha}| \boldsymbol{\rho}_{1}+|\boldsymbol{\beta}| \boldsymbol{\rho}_{2}} M_{k}\left(\frac{\bar{d}\left(Y_{k}, \overline{0}\right)}{\boldsymbol{\rho}_{2}}\right)\right]^{p_{k}} \\
& \leq D K \sum_{k=1}^{\infty} a_{n k}\left[M_{k}\left(\frac{\bar{d}\left(X_{k}, \overline{0}\right)}{\rho_{1}}\right)\right]^{p_{k}}+D K \sum_{k=1}^{\infty} a_{n k}\left[M_{k}\left(\frac{\bar{d}\left(Y_{k}, \overline{0}\right)}{\rho_{2}}\right)\right]^{p_{k}} \\
& \text { where } K=\max \left\{1,\left(\frac{|\alpha|}{|\boldsymbol{\alpha}| \rho_{1}+|\boldsymbol{\beta}| \rho_{2}}\right)^{H},\left(\frac{|\boldsymbol{\alpha}|}{|\boldsymbol{\beta}| \boldsymbol{\rho}_{1}+|\boldsymbol{\beta}| \boldsymbol{\rho}_{2}}\right)^{H}\right\}
\end{aligned}
$$

From the above relation we obtain the following: 


$$
\begin{aligned}
& \left\{n \in N: \sum_{k=1}^{\infty} a_{n k}\left[M_{k}\left(\frac{\bar{d}\left(\boldsymbol{\alpha} X_{k}+\boldsymbol{\beta} Y_{k}, \overline{0}\right)}{|\boldsymbol{\alpha}| \boldsymbol{\rho}_{1}+|\boldsymbol{\beta}| \boldsymbol{\rho}_{2}}\right)\right]^{p_{k}} \geq \boldsymbol{\varepsilon}\right\} \subseteq \\
& \left\{n \in N: D K \sum_{k=1}^{\infty} a_{n k}\left[M_{k}\left(\frac{\bar{d}\left(X_{k}, \overline{0}\right)}{\boldsymbol{\rho}_{1}}\right)\right]^{p_{k}} \geq \frac{\boldsymbol{\varepsilon}}{2}\right\} \\
& \cup\left\{n \in N: D K \sum_{k=1}^{\infty} a_{n k}\left[M_{k}\left(\frac{\bar{d}\left(Y_{k}, \overline{0}\right)}{\boldsymbol{\rho}_{2}}\right)\right]^{p_{k}} \geq \frac{\boldsymbol{\varepsilon}}{2}\right\} \in I
\end{aligned}
$$

This completes the proof.

Theorem 4.2. $w^{I(F)}[A, M, p], w^{I(F)}[A, M, p]_{0}$, and $w^{I(F)}[A, M, p]_{\infty}$ are linear are linear topological space spaces with the paranorm $g$ defined by

$$
g(X)=\inf \left\{\rho^{\frac{p_{n}}{H}}:\left(\sum_{k=1}^{\infty} a_{n k}\left[M_{k}\left(\frac{\bar{d}\left(X_{k}, \overline{0}\right)}{\rho}\right)\right]^{p_{k}}\right)^{1 / H} \leq 1, \text { for some } \rho>0, n=1,2,3, \ldots\right\},
$$

where $H=\max \left\{1, \sup _{k} p_{k}\right\}$.

Proof. This can be easily verified by using standard techniques and so is omitted.

Theorem 4.3. (a) Let $0<\inf p_{k} \leq p_{k} \leq 1$. Then

$$
\begin{aligned}
& w^{I(F)}[A, M, p] \subseteq w^{I(F)}[A, M], w^{I(F)}[A, M, p]_{0} \subseteq w^{I(F)}[A, M]_{0} \\
& \text { (b) Let } 1 \leq p_{k} \leq \sup p_{k}<\infty \text {. Then } \\
& w^{I(F)}[A, M] \subseteq w^{I(F)}[A, M, p], w^{I(F)}[A, M]_{0} \subseteq w^{I(F)}[A, M, p]_{0}
\end{aligned}
$$

Proof. (a) Let $X=\left(X_{k}\right)$ be an element in $w^{I(F)}[A, M, p]$. Since $0<\inf p_{k} \leq p_{k} \leq 1$ we have

Therefore

$$
\sum_{k=1}^{\infty} a_{n k}\left[M_{k}\left(\frac{\bar{d}\left(X_{k}, X_{0}\right)}{\rho}\right)\right] \leq \sum_{k=1}^{\infty} a_{n k}\left[M_{k}\left(\frac{\bar{d}\left(X_{k}, X_{0}\right)}{\rho}\right)\right]^{p_{k}}
$$

$$
\begin{aligned}
& \left\{n \in N: \sum_{k=1}^{\infty} a_{n k}\left[M_{k}\left(\frac{\bar{d}\left(X_{k}, X_{0}\right)}{\rho}\right)\right] \geq \boldsymbol{\varepsilon}\right\} \\
& \subseteq\left\{n \in N: \sum_{k=1}^{\infty} a_{n k}\left[M_{k}\left(\frac{\bar{d}\left(X_{k}, X_{0}\right)}{\rho}\right)\right]^{p_{k}} \geq \boldsymbol{\varepsilon}\right\}
\end{aligned}
$$

The other part can be proved in similar way.

(b) Let $X=\left(X_{k}\right)$ be an element in $w^{I(F)}[A, M, p]$. Since $1 \leq p_{k} \leq$ sup $p_{k}<\infty$. Then for each $0<\varepsilon<1$ there exists a positive integer $n_{0}$ such that 


$$
\sum_{k=1}^{\infty} a_{n k}\left[M_{k}\left(\frac{\bar{d}\left(X_{k}, X_{0}\right)}{\rho}\right)\right] \leq \varepsilon<1 \text { for all } n \geq n_{0}
$$

This implies that

$$
\sum_{k=1}^{\infty} a_{n k}\left[M_{k}\left(\frac{\bar{d}\left(X_{k}, X_{0}\right)}{\rho}\right)\right]^{p_{k}} \leq \sum_{k=1}^{\infty} a_{n k}\left[M_{k}\left(\frac{\bar{d}\left(X_{k}, X_{0}\right)}{\rho}\right)\right]
$$

Therefore we have

$$
\begin{aligned}
& \left\{n \in N: \sum_{k=1}^{\infty} a_{n k}\left[M_{k}\left(\frac{\bar{d}\left(X_{k}, X_{0}\right)}{\rho}\right)\right]^{p_{k}} \geq \boldsymbol{\varepsilon}\right\} \subseteq \\
& \subseteq\left\{n \in N: \sum_{k=1}^{\infty} a_{n k}\left[M_{k}\left(\frac{\bar{d}\left(X_{k}, X_{0}\right)}{\rho}\right)\right] \geq \boldsymbol{\varepsilon}\right\} \in I
\end{aligned}
$$

The other part can be proved in similar way.

Proposition 4.4. The sequence spaces $w^{I(F)}[A, M, p]_{0}$. and $w^{I(F)}[A, M, p]_{\infty}$ are normal as well as monotone.

Proof. We give the proof of the theorem for $w^{I(F)}[A, M, p]_{0}$ only. Let $X=$ $\left(X_{k}\right) \in W^{I(F)}[A, M, p]_{0}$ and $Y=\left(Y_{k}\right)$ be such that $\bar{d}\left(Y_{k}, \overline{0}\right) \leq \bar{d}\left(X_{k}, \overline{0}\right)$ for all $k \in N$. Then forgiven $\varepsilon>0$ we have

$$
B=\left\{n \in N: \sum_{k=1}^{\infty} a_{n k}\left[M_{k}\left(\frac{\bar{d}\left(X_{k}, \overline{0}\right)}{\rho}\right)\right]^{p_{k}} \geq \boldsymbol{\varepsilon}\right\} \in I .
$$

Again the set

$$
B_{1}=\left\{n \in N: \sum_{k=1}^{\infty} a_{n k}\left[M_{k}\left(\frac{\bar{d}\left(Y_{k}, \overline{0}\right)}{\rho}\right)\right]^{p_{k}} \geq \boldsymbol{\varepsilon}\right\} \subseteq B .
$$

Hence $B_{1} \in I$ and so $Y=\left(Y_{k}\right) \in w^{I(F)}[A, M, p]_{0}$. Thus the space $w^{I(F)}[A, M, p]_{0}$ is normal. Also from the Lemma 2.1, it follows that $w^{I(F)}[A, M, p]_{0}$ is monotone. 


\section{REFERENCES}

1. H. Altinok, R. Colak, M. Et, $\lambda$-Difference sequence spaces of fuzzy numbers, Fuzzy Sets and System 160 3128-3139, 2009.

2. B. Hazarika, and E. Savas, Some I -convergent lamda-summable difference sequence spaces of fuzzy real numbers defined by a sequence of Orlicz, Mathematical and Computer Modelling 54, 2986-2998, 2011.

3. O. Kaleva, S. Seikkala, On fuzzy metric spaces, Fuzzy Sets and Systems, 12215-229, 1984.

4. P.K. Kamthan, M. Gupta, Sequence spaces and Series, Marcel Dekker, 1980.

5. M. A. Krasnoselskii, Y. B. Rutitsky, Convex functions and Orlicz functions, Groningen, Netharland, 1961.

6. P. Kostyrko, T. Salat, and W. Wilczynski, I-convergence, Real Analysis Exchange 26(2), 669-686, 2000-2001.

7. V. Kumar, K Kumar, On the ideal convergence of sequences of fuzzy numbers, Information Sciencies 178 4670-4678, 2008.

8. J.S. Kwon, On statistical and p-Cesaro convergence of fuzzy numbers, The Korean journal of computational \& applied mathematics 7 195-203, 2000.

9. J. Lindenstrauss, L. Tzafriri, On Orlicz sequence spaces, Israel Journal of Mathematics 101, 379-390, 1971.

10. M. Matloka, Sequences of fuzzy numbers, BUSEFAL 28 28-37, 1986.

11. M. Mursaleen, M. Basarir, On some new sequence spaces of fuzzy numbers, Indian Journal of Pure and Applied Mathematics 34(9) 1351-1357, 2003.

12. S. Nanda, On sequences of fuzzy numbers, Fuzzy Sets and Systems 33 123-126, 1989.

13. F. Nuray, S. Savas, Statistical convergence of sequences of fuzzy numbers, Mathematica Slovaca 45 269-273, 1995.

14. S. D. Parashar, B. Choudhury, Sequence space defined by Orlicz functions, Indian Journal of Pure and Applied Mathematics, 25(14) 419-428,1994.

15. M.L. Puri and D. A. Ralescu, Differential of fuzzy numbers, Journal of Mathematical Analysis and Applications 91 552-558, 1983.

16. W. H. Ruckle, FK-spaces in which the sequence of coordinate vectors is bounded, Canadian Journal of Mathematics 25 973-978,1973.

17. E. Savas, A note on double sequence of Fuzzy numbers, Turkish Journal of Mathematics, 20 175-178, 1996.

18. E. Savas, $\Delta \mathrm{m}$-strongly summable sequences spaces in 2-Normed Spaces defined byIdeal Convergence and an Orlicz Function, Applied Mathematics and Computation 217, 271-276, 2010.

19. E. Savas, A-sequence spaces in 2-normed space defined by ideal convergence and anOrlicz function, Abstract and Applied Analysis, Vol. 2011, Article ID 741382, 2011.

20. E. Savas, On some new sequence spaces in 2-normed spaces using Ideal convergence and an Orlicz function, About Journal of Inequalities and Applications, Article Number: 482392 DOI:10.1155/2010/482392, 2010.

21. E. Savas and Mursaleen, On statistically convergent double sequences of fuzzy numbers, Information Sciences 162, 3-4183-192, 2004. 
22. E. Savas, A note on sequence of Fuzzy numbers, Information Sciences 124, 297 300, 2000.

23. E. Savas, On strongly $\lambda$-summable sequences of Fuzzy numbers, Information Sciences 125, 181-186, 2000.

24. E. Savas, On statistically convergent sequence of Fuzzy numbers, Information Sciences 137, 272-282, 2001.

25. E. Savas, Onlacunary statistical convergent sequences of fuzzy numbers, Applied Mathematics Letters 21, 134-141 2008.

26. E. Savas and Richard F. Patterson, An Orlicz extension of some new sequencespaces, Rend. Istit. Mat. Univ. Trieste 37, no. 1-2,145-154, 2006.

27. E. Savas and R. Savas, Some sequence spaces defined by Orlicz functions, Archiv der Mathematik (Brno) 40, 33-40, 2004.

28. E. Savas, R. F. Patterson, Some double lacunary sequence spaces defined by Orliczfunctions, Southeast Asian Bulletin of Mathematics, 35(1), 103-110, 2011.

29. E. Savas, On some new double lacunary sequences spaces via Orlicz function, Journal of Computational Analysis and Applications, 11(3), 423-430, 2009.

30. E. Savas, Pratulananda Das, A generalized statistical convergence via ideals, Applied Mathematics Letters 24 826-830, 2011.

31. E. Savas, Pratulananda Das, S. Dutta, A note on strong matrix summability viaideals, Applied Mathematics Letters 25 (4), 733-738, 2012.

32. E. Savas, On generalized double statistical convergence via ideals, The Fifth Saudi Science Conference, 16-18 April, 2012. 